\section{Further development and evaluation of a breast/ovarian cancer genetics referral screening tool}

\section{To the Editor:}

This letter is being written in follow-up to our original article "Evaluation of a breast/ovarian cancer genetics referral screening tool in a mammography population" published in the November 2009 issue of Genetics in Medicine. ${ }^{1}$ The tool is designed to quickly identify individuals who are appropriate to refer for cancer risk consultation regarding $B R C A 1 / 2$ testing.

We modified the original paper-based tool in an attempt to address some of its limitations and have created an interactive web version (breast cancer genetics referral screening tool [BRST]) to enhance accessibility. The tool now distinguishes between maternal and paternal lineages when identifying second-degree relatives. We have added nieces and nephews to the list of included second-degree relatives. In addition, the parameters of bilateral breast cancer, and breast and ovarian cancer in the same individual, have been added to the algorithm.

We evaluated the new web-based version of the tool using the pedigree data collected on the subjects in the original study. ${ }^{1}$ As before, the tool score (positive or negative) was compared with analysis of the full pedigrees using BRCAPRO, Myriad II, BOADICEA, and family history assessment tool (FHAT), with $\mathrm{a} \geq 10 \%$ BRCA1/2 mutation probably or an FHAT score of 10 as the true measure of "high" risk. Table 1 provides the validation statistics of the revised tool (Table 1). Sensitivity increased for all model comparisons $(+8.2 \%$ to $+11.1 \%)$, whereas specificity decreased slightly $(-0.4 \%$ to $-2.3 \%)$. As hypothesized in the original article, the addition of bilaterality accounted for the majority of the reduction in specificity. How-

\begin{tabular}{lccccc}
\hline Table 1 & B-RST validation measures & & \\
\hline Measure & BOADICEA & BRCAPRO & Myriad II & FHAT & Overall $^{a}$ \\
\hline Sensitivity (\%) & 100 & 100 & 96.9 & 90.0 & 89.4 \\
Specificity (\%) & 75.1 & 74.0 & 76.1 & 89.8 & 91.5 \\
ROC AUC & 0.88 & 0.87 & 0.87 & 0.90 & 0.90 \\
$(95 \%$ CI) & $(0.83-0.92)$ & $(0.83-0.91)$ & $(0.81-0.92)$ & $(0.85-0.94)$ & $(0.85-0.95)$
\end{tabular}

${ }^{a}$ High risk by at least one model $=$ actual positive state.

B-RST, breast cancer genetics referral screeningtool; ROC AUC, area under the receiver operator characteristic curve; CI, confidence interval.

ever, we chose to retain this parameter in the web version, because it is included in most BRCA1/2-related guidelines and models, and maximizing sensitivity is desired.

The revised tool (B-RST) is now available (open access) on the web at: http://www.brcagenscreen.org. There is both a health care provider and a consumer portal. Information entered by users is not stored, but a printable report is provided, which includes the screen result, interpretation, disclaimers, family history survey responses, and resources. Future studies to access the clinical utility of the tool are planned.

Cecelia Bellcross, MS, PhD

Office of Public Health Genomics Centers for Disease Control and Prevention Atlanta, Georgia

Disclosure: The author declares no conflict of interest.

\section{REFERENCE}

1. Bellcross C, Lemke A, Pape L, Tess AL, Meisner LT. Evaluation of a breast/ovarian cancer genetics referral screening tool in a mammography population. Genet Med 2009;11:783-788. 